\title{
Indicaciones de artroplastia en lesiones del mango rotador
}

\author{
Indications for arthroplasty in rotator cuff injuries
}

\author{
César Ernesto Millán Osuna, ${ }^{*}$ Roberto Porras González, ${ }^{\ddagger}$ Alfonso Chávez Valenzuela, ${ }^{\S}$ \\ Jonathan Achoy Insunza," Michell Ruiz Suárez"
}
* Cirujano Ortopedista. Egresado del Instituto Nacional de Rehabilitación. Alta Especialidad
en Traumatología Deportiva. Médico adscrito del Hospital Ángeles Tijuana.
‡ Cirujano Ortopedista. Egresado del Hospital Regional de Alta Especialidad de Veracruz. Alta
Especialidad en Traumatología Deportiva. Médico adscrito del Hospital Ángeles León.
$\S$ Cirujano Ortopedista. Egresado del Hospital Universitario de Puebla. Fellow
Artroscopia y Reconstrucción articular del Hospital Ángeles Tijuana.
॥ Cirujano Ortopedista. Egresado del Hospital Civil de Culiacán. Fellow Artroscopia
y Reconstrucción articular del Hospital Ángeles Tijuana.
" Cirujano Ortopedista. Egresado del Instituto Nacional de Rehabilitación. Alta Especialidad en
Cirugía de Hombro y Codo. Médico adscrito del Hospital Ángeles Metropolitano.

Correspondencia: Dr. César Ernesto Millán Osuna. Paseo de los Héroes No. 10999-301, Zona Río, 22010, Tijuana, Baja California. Correo electrónico: drmillanosuna.orto@gmail.com

\section{RESUMEN}

Las lesiones masivas del manguito de los rotadores son patologías cada vez más frecuentes, las cuales pueden ocasionar una disfunción severa del hombro, ya que desarrollan una artropatía y/o pseudoparálisis del mismo. Hoy en día hay confusión en cuanto a la definición de la pseudoparálisis; sin embargo, contamos con literatura que nos ayuda a identificar con mayor precisión esta problemática, ya que es crucial para llevar a cabo un buen manejo terapéutico. Nos queda claro con base en las clasificaciones de Goutallier y Patte cuáles son las lesiones masivas del mango rotador que no son candidatas a reparación, con lo cual coloca a la artroplastia de hombro como una alternativa viable para la resolución de esta patología. Existen reportes donde se fundamenta por qué no se sigue indicando la artroplastia total de hombro en la artropatía y/o pseudoparálisis del hombro, y se propone el uso de la hemiprótesis tipo

\section{ABSTRACT}

Massive rotator cuff injuries are increasingly frequent pathologies, which can cause severe shoulder dysfunction, developing arthropathy and/or pseudoparalysis of the shoulder. Nowadays there is confusion regarding the definition of pseudoparalysis, however, there is literature that helps us identify this problem more precisely, since it is crucial to carry a good treatment. It is clear to us based on the Goutallier and Patte classifications, which are the massive rotator cuff injuries that are not candidates for repair; which leaves us with shoulder arthroplasty as a viable alternative for the resolution of this pathology. There are reports where it is justified why total shoulder arthroplasty is not indicated in arthropathy and/or pseudoparalysis of the shoulder, and the use of CTA-type hemiprosthesis or the reverse prosthesis is proposed as the solution, however, we must take into account the age, clinic, activity level and the expectation of the 
CTA o la prótesis reversa como la solución; sin embargo, para ello debemos tomar en cuenta la edad, clínica, nivel de actividad y la expectativa del paciente a tratar. Hacemos una breve revisión de la literatura, la cual compartimos con la finalidad de ayudar al ortopedista a realizar una mejor elección del manejo de las lesiones irreparables del manguito rotador.

Palabras clave: Lesión masiva del manguito rotador, artropatía de hombro, pseudoparálisis, hemiartroplastia, artroplastia reversa. patient to be treated. We do a brief review of the literature and share it with you in order to help the orthopedic surgeon make a better choice in the management of irreparable rotator cuff injuries.

Keywords: Massive rotator cuff injury, shoulder arthropathy, pseudoparalysis, hemiarthroplasty, reverse arthroplasty.

El manguito de los rotadores está formado por cuatro músculos y sus respectivas porciones tendinosas que se insertan en la cabeza humeral, los cuales ayudan al movimiento, rotación y estabilidad del hombro, mediante la centralización de la cabeza humeral en la cavidad glenoidea. La interacción del músculo deltoides y el manguito rotador desempeña un rol importante en la estabilidad dinámica del hombro, donde el mango rotador ejerce una fuerza inferior y de compresión sobre la cabeza humeral, mientras que el deltoides provee una fuerza superior, resultando en un balance de fuerza o acoplamiento de fuerza.

Una lesión del manguito rotador provoca dolor y disfunción del hombro. La prevalencia del dolor de hombro en la población general oscila entre 4 y $39 \%,{ }^{2}$ la cual se relaciona con trastornos del manguito rotador principalmente en adultos mayores. La incidencia de las lesiones del manguito rotador se observa de 7 a $20 \%$ en las lesiones de espesor completo en estudios en cadáveres, llegando hasta $30 \%$ si se incluyen las lesiones de espesor parcial. ${ }^{3}$

Hay diversas lesiones del manguito rotador que pueden ser secundarias a eventos traumáticos, degenerativos o son consecuencia de enfermedades inflamatorias. Se encuentran en alrededor de $15-20 \%$ en las personas de 60 años de edad, de $26-30 \%$ en las de 70 años y de $36-50 \%$ en las de 80 años. Ha aumentado la frecuencia de estas lesiones en jóvenes debido a acciones asociadas a movimientos repetitivos que realizan por encima de la cabeza, como el béisbol, y también por ejercicios como levantamiento de pesas. ${ }^{4}$

Se han propuesto múltiples clasificaciones de lesiones masivas del manguito rotador. Actualmente se definen como una lesión con al menos dos tendones rotos o rotura del espesor completo del tendón del supraespinoso de una longitud superior a $5 \mathrm{~cm} .{ }^{5}$ Las lesiones masivas siempre están asociadas a pérdida de fuerza y dolor incapacitante, rara vez se deben a un evento agudo, generalmente son crónicas y están asociadas a retracción miotendinosa, pérdida de elasticidad musculotendinosa, infiltración grasa muscular, subluxación superior de la cabeza humeral y osteoartritis. Si la infiltración grasa de los músculos respectivos se observa más allá de la etapa 2 de Goutallier, o si hay migración cefálica del húmero que resulte en una distancia acromiohumeral de menos de $7 \mathrm{~mm}$, la probabilidad de que la reparación del manguito sea exitosa es muy baja, por lo que se considera una lesión irreparable. ${ }^{6}$ 
La resonancia magnética es el método diagnóstico de elección en las roturas masivas del manguito rotador, con una sensibilidad de $89 \%$ y una especificidad de $100 \%,{ }^{7}$ con la cual podemos valorar el grado de lesión así como obtener un pronóstico y un tratamiento de acuerdo con las clasificaciones de Goutallier y Patte.

La clasificación de Goutallier se refiere al grado de infiltración grasa, la cual es un factor de mal pronóstico para la reparación de los tendones del manguito (Tabla 1). ${ }^{8}$

A la vez, Patte elabora su clasificación de acuerdo a la retracción coronal del tendón. Las características a evaluar en una rotura son: los tendones afectados, el tamaño de la rotura, y si la afectación comprende el espesor total o parcial del tendón (Tabla 2). ${ }^{9}$

En 1983 Neer y colaboradores describen el término de artropatía del manguito rotador como una alteración de la articulación glenohumeral secundaria a una lesión masiva del manguito, lo cual implica una insuficiencia del manguito rotador, ascenso de la cabeza humeral, alteración y pérdida de líquido sinovial, destrucción del cartílago articular, acetabulización acromial, aplanamiento del troquíter y osteopenia, ${ }^{10}$ lo que propicia la pérdida de compresión de la cabeza humeral con la glenoides, resultando una alteración en la cinemática del hombro. Esta patología se presenta más frecuentemente en mujeres entre la sexta y séptima década de la vida, el lado dominante es el más afectado, se manifiesta por dolor crónico progresivo, crepitación y disminución del rango de movilidad. ${ }^{11}$

La clasificación de la artropatía del manguito rotador incluye el sistema de Hamada (Tabla 3) y el sistema Seebauer. La clasificación de Hamada detalla las diferentes etapas de la lesión masiva del manguito rotador detectadas en radiografía, donde cada grado demuestra cambios en la progresión de la artropatía; mientras que la clasificación de Seebauer se refiere a una descripción biomecánica de la artropatía, donde cada estadio se distingue por el grado de migración superior del centro de rotación y de inestabilidad, dividiéndose en cuatro estadios: IA articulación estable con mínima migración debido a una contención intacta con la presencia de acetabulización del arco coracoacromial y femoralización de la cabeza humeral. IB agrega la presencia de erosión medial de la glenoides que compromete la estabilidad de la articulación, pero se mantiene contenida. IIA se observa con traslación superior de la cabeza humeral y migración del centro de rotación de la articulación por pérdida de contención anterior, se mantiene mínimamente estable por la presencia del arco coracoa-

\begin{tabular}{cccccc}
\hline \multicolumn{7}{c}{ Tabla 1: Clasificación de Goutallier. } \\
\hline $\begin{array}{c}\text { Hallazgos en } \\
\text { tomografía }\end{array}$ & $\begin{array}{c}\text { Músculo } \\
\text { completamente } \\
\text { normal, sin } \\
\text { ninguna raya } \\
\text { grasa }\end{array}$ & $\begin{array}{c}\text { El músculo } \\
\text { contiene algún } \\
\text { trazo de infiltra- } \\
\text { ción grasa }\end{array}$ & $\begin{array}{c}\text { La infiltración } \\
\text { grasa es impor- } \\
\text { tante, pero hay } \\
\text { más músculo } \\
\text { que grasa }\end{array}$ & $\begin{array}{c}\text { Cantidades } \\
\text { iguales de grasa } \\
\text { y músculo }\end{array}$ & $\begin{array}{c}\text { Hay más grasa } \\
\text { que músculo }\end{array}$ \\
\hline
\end{tabular}


cromial. IIB se presenta con escape anterosuperior de la cabeza humeral por pérdida de su contención anterior del arco coracoacromial. ${ }^{12,13}$

Una gran problemática a la cual nos seguimos enfrentando y que representa un reto para el ortopedista es la pseudoparálisis del hombro, la cual se encuentra relacionada con las lesiones del manguito rotador. Este término no tiene una definición uniforme, hay una gran confusión entre pseudoparálisis y pseudoparesia. ${ }^{14}$ Parálisis significa que no hay movimiento y paresia significa debilidad con algo de movimiento. La definición suele confundirse con dolor de hombro, por lo cual la pseudoparálisis aparente debe descartarse al poder eliminar el dolor sólo con manejo analgésico. ${ }^{15}$ Por lo anterior, se define pseudoparálisis como la rotura masiva del manguito rotador, $0^{\circ}$ de elevación activa y elevación pasiva completa, generalmente con escape anterosuperior, dolor que se elimina con la inyección de lidocaína La pseudoparesia se define como la rotura masiva del manguito rotador, elevación activa $<90^{\circ}$, elevación pasiva completa, sin escape anterosuperior y dolor que desaparece con inyección de lidocaína. ${ }^{16}$ Las bases biomecánicas de esta causa se cree que son debido a una insuficiencia en la centralización de la cabeza humeral en la cavidad glenoidea por insuficiencia del manguito rotador, ocasionando una subluxación anterosuperior de la cabeza humeral por la tracción superior del deltoides al intento de elevación, a la vez, se le atribuye al tamaño y ubicación de la rotura completa del supraespinoso y subescapular, o la rotura de tres tendones. ${ }^{17}$

Se han definido los factores pronósticos favorables para la reparación de las rupturas del manguito rotador:

1. Lesión circunscrita a la afectación de un solo tendón.

2. Ausencia de atrofia o degeneración grasa de los músculos del manguito.

3. Distancia acromio humeral $>6 \mathrm{~mm}$.

4. Poco tiempo transcurrido desde la lesión.

5. Pacientes menores de 65 años. ${ }^{18}$

La artroplastia de hombro ha probado ser un tratamiento efectivo para patologías degenerativas, traumáticas, enfermedades inflamatorias y en artropatía del manguito rotador y/o pseudoparálisis una vez que la reparación del manguito rotador no sea una opción viable. Neer y colaboradores propusieron en 1982 la prótesis total de hombro como una alternativa para las insuficiencias del manguito rotador, comentando que la estabilidad de la prótesis depende del 
tamaño y versión de los componentes, y que la función depende de la rehabilitación del mango rotador y del deltoides. ${ }^{19}$ Para limitar la subluxación superior del componente humeral se ha utilizado un componente glenoideo semiconstreñido o una prótesis no constreñida con reparación del manguito. En 1988 Franklin y su equipo asociaron el aflojamiento del componente glenoideo con la insuficiencia del manguito de los rotadores, lo cual condiciona a una subluxación superior del componente humeral, la imagen radiográfica que observan en dicho aflojamiento glenoideo la denominan rocking horse o caballo mecedora..$^{20}$ Más recientemente Nwakama y colegas reportaron $85 \%$ de resultados no favorables en pacientes con artrosis glenohumeral y lesión masiva del manguito rotador tratados con artroplastia total semiconstreñida de hombro, por lo cual se concluye la contraindicación de la artroplastia total de hombro como tratamiento para la artropatía del manguito rotador. ${ }^{21}$

La hemiartroplastia de hombro sigue siendo una opción viable para las lesiones irreparables del manguito rotador sin pseudoparálisis de hombro, con esto se elimina el riesgo de aflojamiento del componente glenoideo. Se han reportado buenos resultados con la hemiprótesis en la flexión activa y disminución del dolor en pacientes con lesiones irreparables del manguito rotador, siempre y cuando se conserve preoperatoriamente un movimiento pasivo completo, función normal del deltoides y artrosis glenohumeral leve. Pollock y colaboradores reportan una disminución similar en cuanto al dolor en pacientes con artropatía del manguito rotador tratados con artroplastia total de hombro vs. hemiartroplastia; sin embargo, reportan mejores resultados en los rangos de movilidad y en la realización de actividades de la vida diaria en los pacientes mayores en las hemiartroplastias (tipo CTA) que son una opción actual, una vez que se descarte la pseudoparálisis. ${ }^{22}$ Las hemiprótesis CTA permiten el apoyo de la cabeza en la glenoides y acromion mejorando la estabilidad del implante y el brazo de palanca del deltoides y con esto el movimiento del hombro, siempre y cuando no exista compromiso de la estabilidad glenohumeral anterosuperior, que se presente ligero desgaste articular glenoideo, y que persista un arco acromioclavicular y un deltoides funcional; ${ }^{23}$ sin embargo, se han reportado ciertas complicaciones, ya que existe un riesgo de pérdida ósea de la glenoides y el acromion (Figura 1), inestabilidad humeral, y una limitada mejoría de la movilidad del hombro. Sánchez-Sotelo y su equipo reportaron inestabilidad anterosuperior en siete de cada 30 pacientes y sólo $67 \%$ de éxito a cinco años de haberse realizado la cirugia. ${ }^{13,24}$

Paul Grammont diseñó en 1987 una nueva prótesis reversa de hombro, cuya principal característica era la medialización del centro de rotación. ${ }^{25}$ Al estar el centro de rotación sobre la

\begin{tabular}{|c|c|}
\hline Grado & \\
\hline 1 & Intervalo acromio humeral mayor de $6 \mathrm{~mm}$ \\
\hline 2 & $\begin{array}{l}\text { Intervalo acromio humeral igual o menor } \\
\text { de } 5 \mathrm{~mm}\end{array}$ \\
\hline 3 & Acetabulización del acromion \\
\hline 4 & $\begin{array}{l}\text { Grado } 3 \text { más disminución del espacio } \\
\text { glenohumeral }\end{array}$ \\
\hline 5 & $\begin{array}{l}\text { Colapso de la cabeza humeral, estadio } \\
\text { final de la artropatía secundaria a rotura del } \\
\text { manguito de los rotadores }\end{array}$ \\
\hline
\end{tabular}


glena directamente, las fuerzas de torsión que se aplicaban sobre la metaglena-glenósfera eran mejor absorbidas que con los modelos previos. Esta prótesis denominada Delta III ha sido la base del desarrollo actual de las prótesis reversas. Grammont reportó la indicación clínica de su prótesis y los primeros resultados, y determinó que la indicación principal era para las artropatías del mango rotador con un deltoides funcional.

La artropatía del mango rotador sigue siendo la indicación principal de la prótesis reversa (Figura 2), por eso el ortopedista debe hacer una adecuada selección del paciente que es candidato a este tipo de artroplastia, haciendo un estudio completo con la exploración física, edad, antecedentes clínicos y estudios de imagenología del paciente. La artropatía del mango rotador con ausencia de rotación externa preoperatoria condicionará una mejoría funcional postquirúrgica en la elevación anterior, pero no en la rotación externa. Los casos más difíciles de tratar son sin duda los pacientes con artropatía del manguito con edad menor de 65 años y con pseudoparálisis. En estos casos habrá que decidir entre una hemiartroplastia a expensas de sacrificar función, o colocar una prótesis reversa asumiendo que pueda haber un deterioro de la función con el paso de los años. Sirveaux y colaboradores reportan $96 \%$ de dolor mínimo o ausencia de dolor en pacien-
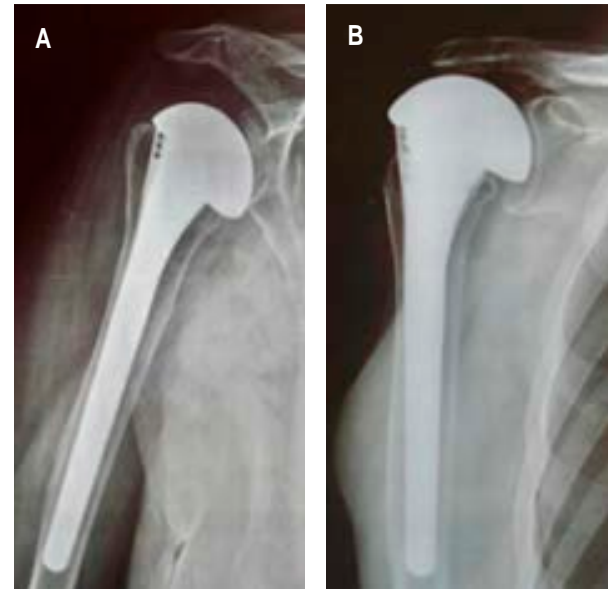

Figura 1: A) Hemiprótesis con cabeza CTA en insuficiencia del manguito rotador. B) Hemiprótesis con cabeza CTA con migración superior y erosión del acromion. Cortesía Dr. Michell Ruiz.

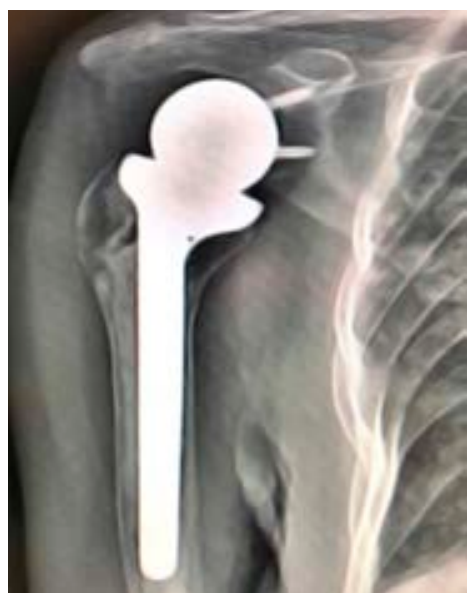

Figura 2: Artroplastia reversa de hombro en paciente con lesión irreparable del manguito rotador y pseudoparálisis. Cortesía Dr. Michell Ruiz. tes con osteoartritis y lesión masiva del manguito rotador tratados con artroplastia reversa de hombro, a la vez refieren mejoría notable en la elevación anterior del hombro. ${ }^{26}$ Young y su equipo también reportan de buenos a excelentes resultados hasta en $89 \%$ de los casos con pocas complicaciones en un seguimiento promedio de 38 meses. ${ }^{27}$ Por su parte Frankle y colegas publican una mejoría significativa en flexión y abducción. Las revisiones de este modelo protésico y sus complicaciones se 
han llegado a reportar en 26 y $71 \%$ respectivamente; ${ }^{13}$ sin embargo, continúa siendo una muy buena alternativa para el tratamiento de las lesiones masivas del manguito rotador con pseudoparálisis de hombro, que logra bajar las cifras de las revisiones y complicaciones con la mejoría en cuanto a la técnica quirúrgica y la experiencia del ortopedista.

\section{CONCLUSIÓN}

Actualmente diagnosticamos con mayor frecuencia las lesiones masivas del manguito rotador irreparables, ya que ha aumentado la expectativa de vida en la población, obligándonos a tener conocimiento del manejo de estas patologías. Existen diversos métodos de tratamiento, entre los cuales nos debe quedar claro que la artroplastia total de hombro está contraindicada y la hemiatroplastia con cabeza grande está indicada en los pacientes sin pseudoparálisis agregada; asimismo debe valorarse el uso de artroplastia reversa de hombro en quienes presenten pseudoparálisis.

\section{BIBLIOGRAFÍA}

1. Burkhart SS. Flouroscopic comparison of kinematic patterns in massive rotator cuff tear A suspension bridge model. Clin Orthop Relat Res. 1992; (284): 144-152.

2. Langley PC, Ruiz-Iban MA, Molina JT, De Andres J, Castellón JR. The prevalence, correlates and treatment of pain in Spain. J Med Econ. 2011; 14 (3): 367-380.

3. Reilly P, Macleod I, Macfarlane R, Windley J, Emery RJ. Dead men and radiologists don't lie: a review of cadaveric and radiological studies of rotator cuff tear prevalence. Ann R Coll Surg Engl. 2006; 88 (2): 116-121.

4. Rashid M, Cooper C, Cook J, Cooper D, Dakin S, Snelling S, et al. Increasing age and tear size reduce rotator cuff repair healing rate at 1 year. Acta Orthop. 2017; 88: 1-6.

5. Hansen ML, Otis JC, Johnson JS, Cordasco FA, Craig EV, Warren RF. Biomechanics of massive rotator cuff tear: implications for treatment. J Bone Joint Surg Am. 2008; 90 (2): 316-325.

6. Gerber C, Wirth SH, Farshad M. Treatment options for massive rotator cuff tears. J Shoulder Elbow Surg. 2011; 20 (2): S20-29.

7. Iannotti JP, Zlatkin MB, Esterhai JL, Kressel HY, Dalinka MK, Spindler KP. Magnetic resonance imaging of shoulder. Sensitivity, specificity, and predictive value. J Bone Joint Surg Am. 1991; 73 (1): 17-29.

8. Goutallier D, Postel JM, Bernageau J, Lava L, Voisin MC. Fatty muscle degeneration in cuff ruptures. Pre-and postoperative evaluation by CT scan. Clin Orthop Relat Res. 1994; (304): 78-83.

9. Patte D. Classification of rotator cuff lesions. Clin Orthop Relat Res. 1990; (254): 81-86.

10. Neer CS 2nd, Craig EV, Fukuda H. Cuff-tear arthropathy. J Bone Joint Surg Am. 1983; 65 (9): 12321244.

11. Carvalho CD, Andreoli CV, Pochini AC, Ejnismann B. Use of cuff tear arthroplasty head prosthesis for rotator cuff arthropathy treatment in elderly patients with comorbidities. Einstein. 2016; 14 (4): 520-527.

12. Hamada K, Fukuda H, Mikasa M, Kobayashi Y. Roentgenographic findings in massive rotator cuff tears. A long-term observation. Clin Orthop Relat Res. 1990; (254): 92-96.

13. Nam D, Maak TG, Raphael BS, Kepler CK, Cross MB, Warren RF. Rotator cuff tear arthropathy: Evaluation, diagnosis, and treatment. J Bone Joint Surg Am. 2012; 94 (6): e34.

14. Werner CM, Steinmann PA, Gilbart M, Gerber C. Treatment of painful pseudoparesis due to irreparable rotator cuff dysfunction with the Delta III reverse-ball-and-socket total shoulder prosthesis. J Bone Joint Surg Am. 2005; 87 (7): 1476-1486.

15. Bouaicha S, Ernstbrunner L, Jud L, Meyer DC, Snedeker JG, Bachmann E. The lever arm ratio of the rotator cuff to deltoid muscle explains and predicts pseudoparalysis of the shoulder. Bone Joint J. 2018; 100-B (12): 1600-1608.

16. Tokish JM, Alexander TC, Kissenberth MJ, Hawkins RJ. Pseudoparalysis: a systematic review of term definitions, treatment approaches, and outcomes of management techniques. J Shoulder Elbow Surg. 2017; 26 (6): e177-e187. 
17. Collin P, Matsumura N, Lädermann A, Denard PJ, Walch G: Relationship between massive chronic rotator cuff tear pattern and loss of active shoulder range of motion. J Shoulder Elbow Surg. 2014; 23 (8): 1195-1202.

18. Woerthler K, Waldt S: MR imaging in sports-related glenohumeral instability. Eur Radiol. 2006 ; 16 (12): 2622-2636.

19. Neer CS 2nd, Watson KC, Stanton FJ: Recent experience in total shoulder replacement. J Bone Joint Surg Am. 1982; 64 (3): 319-337.

20. Franklin JL, Barret WP, Jackins SE, Matsen FA 3rd. Glenoid loosening in total shoulder arthroplasty. Association with rotator cuff deficiency. J Arthroplasty. 1988; 3 (1): 39-46.

21. Nwakama AC, Cofield RH, Kavanagh BF, Loehr JF. Semiconstrained total shoulder arthroplasty for glenohumeral arthritis and massive rotator cuff tearing. J Shoulder Elbow Surg. 2000; 9 (4): 302-307.

22. Pollock RG, Deliz ED, Mcllveen SJ, Flatow EL, Bigliani LU. Prosthetic replacement in rotator cuff-deficient shoulders. J Shoulder Elbow Surg. 1992; 1 (4): 173-186.

23. Zuckerman JD, Scott AJ, Gallagher MA. Hemiarthroplasty for cuff tear arthropathy. J Shoulder Elbow Surg. 2000; 9 (3): 169-172.

24. Sanchez-Sotelo J, Cofield RH, Rowland CM. Shoulder hemiarthroplasty for glenohumeral arthritis associated with severe rotator cuff deficiency. J Bone Joint Surg Am. 2001; 83 (12): 1814-1822.

25. Boileau P, Waltkinson DJ, Hatzidakis AM, Balg F. Grammont reverse prosthesis: design, rationale, and biomechanics. J Shoulder Elbow Surg. 2005; 14 (1-S): 147S-161S.

26. Sirveaux F, Favard L, Oudet D, Huquet D, Walch G, Molé D. Grammont inverted total shoulder arthroplasty in the treatment of glenohumeral osteoarthritis with massive rupture of the cuff. Results of a multicentre study of 80 shoulders. J Bone Joint Surg Br. 2004; 86 (3): 388-395.

27. Young SW, Everts NM, Ball CM, Astley TM, Poon PC. The SMR reverse shoulder prosthesis in the treatment of cuff-deficient shoulder conditions. J Shoulder Elbow Surg. 2009; 18 (4): 622-626.

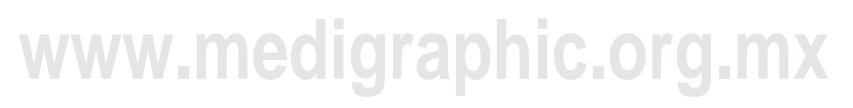

